\title{
Fine images from NTT
}

\section{Garching, West Germany}

FIRST results from the New Technology Telescope (NTT) of the European Southern Observatory (ESO) in La Silla. Chile, bode well for its new 'active optics' technology and for the success of ESO's next big project, the Very Large Telescope (VLT). An inaugural ceremony was held on 6 February at ESO headquarters in Garching to release the good news.

The active optics system relies on 75 servos that can slightly flex the $24-\mathrm{cm}$ thick, 3.6-metre diameter primary mirror of the NTT. The servos are controlled by an image analyser, which looks at 'raw' star images to determine how the mirror shape should be adjusted to compensate for the varying distortions introduced by the passage of light through the Earth's atmosphere.

In good conditions. NTT can resolve star images down to 0.3 arc-seconds, a factor of three better than a conventional 3.6-metre at the La Silla site, in the Chilean Andes, typically achieves.

NTT's success is important for the development of the VLT, an array of four linked 8-metre telescopes to be built in Chile in the mid-1990s. VLT's mirrors will be thinner than NTT's, allowing more rapid adjustment of the mirror profile and therefore even higher resolution. Eventually, NTT will also be fitted with adaptive optics, a faster version of active optics (see Nature 341. 675; 1989).

In an inaugural observing run, done before the telescope had been fully optimized, NTT resolved stars down to 25th magnitude in ten minutes; ESO astronomer Richard West said that 27thmagnitude stars are expected to be resolved in a one-hour exposure.

NTT reveals the detailed structures of galaxies and globular star clusters with unprecedented clarity. A five-minute exposure on 3 December 1989 resolved individual stars in a globular cluster asso-

\section{IMAGE UNAVAILABLE FOR COPYRIGHT REASONS}

FIG. 1 The Fornax dwarf galaxy - the first time a cluster so far away has been fully resolved. ciated with the Fornax dwarf galaxy, 700,000 light-years away, the first time such a distant cluster had been fully resolved (see Fig. 1). Another project will be a search for Cepheids, variable stars whose characteristic brightness changes allow their distance to be determined, in far-off galaxies.

Finding Cepheids is an essential step in estimating the Hubble constant, which measures the rate of expansion of the Universe.

On a night with relatively poor seeing conditions, the NTT was used to image Comet Austin, which is expected to become visible in the Northern Hemisphere by mid-April 1990. A hot spot was seen in the nucleus of the comet, even though it had not yet begun to develop a tail. This was the first time such a feature had been observed on a comet so far from the Sun. (see Fig. 2) Another innovation on NTT will be the use of a satellite links between La Silla and Garching, which will allow astronomers to direct the telescope without leaving Europe. This new system will allow flexibility in scheduling telescope time that was never possible when research teams had fixed stays in faraway Chile. The NTT is already a popular instrument: only a few observing slots are still available this year, after 1 October, and the deadline for proposals is 15 April.

\section{IMAGE UNAVAILABLE FOR COPYRIGHT REASONS}

FIG. 2 Comet Austin - computer enhanced photo showing curves of equal brightness

NTT has already inspired imitators. Italy is building a 3.5-metre telescope called Galileo that is modelled on NTT. (Italy and Switzerland financed a large proportion of NTT when they joined ESO and paid the entry fee in 1982.) Other groups from Australia, South Africa and the United States, as well as several groups from Europe, have expressed interest in purchasing the engineering knowhow for NTT

Speaking at the inauguration ceremony, West German Research Minister Heinz Riesenhuber praised ESO for delivering NTT on time and within its budget of DM 24 million ( $\$ 14$ million). He said that the same kind of efficiency will be necessary to ensure the successful completion of VLT.

Steven Dickman

\section{JAPANESE ELECTION}

\section{US increases pressure on trade issues}

\section{Tokyo}

THE comfortable win for Japan's ruling Liberal Democratic Party (LDP) in the general election on 18 February is expected to open the way for increased US pressure on Japan over science-related trade issues.

The United States had left several contentious trade issues to simmer while the LDP struggled to regain its foothold on power after a series of money and sex scandals brought a crushing defeat for the party in last year's upper house election. The United States clearly views the LDP as preferable to the Japan Socialist Party (JSP), which has traditionally opposed the US-Japan security treaty.

Among the issues that are likely to reemerge are trade in satellites and supercomputers. The United States has been pressing the Japanese government to buy more US supercomputers for years but nearly all public sector orders still go to Japanese companies despite the fact that the US company Cray Research Inc. is the acknowledged leader in this field. Ironically, Japanese private companies such as Toyota and Toshiba are among Cray's best customers. But very few universities and government research institutes have Crays.
Another bone of contention is a couple of next-generation communication satellites (CS-4) due to be developed by the Science and Technology Agency, the Ministry of Post and Telecommunications, and the National Space Development Agency with substantial financial backing from Nippon Telegraph and Telephone (NTT), Japan's semi-private domestic telecommunications giant. Total development costs are estimated at $¥ 90,000$ million ( $\$ 650$ million), 75 per cent of which is to be put up by NTT.

The United States claims that the satellites are a commercial project that should be open to foreign competition, in particular US satellite manufacturers which can offer much cheaper satellites off the shelf to do the same job. But Japan insists that CS-4 is not commercial.

The United States is threatening to invoke the Super 301 provisions of the US 1988 trade act over this and other issues. As a compromise, Japan is expected to suggest at a Japan-US satellite conference later this month that one of the two CS-4 satellites should be bought from overseas and the other built with some foreign procurement of parts and without the financial backing of NTT.
David Swinbanks 